\title{
SALIVA COMO MUESTRA PARA LA DETECCIÓN DE
} SARS-COV-2

\author{
Saliva as a sample for the detection of SARS-CoV-2
}

Antony Orozco-Barquero ${ }^{1}$ Ana Lucía Chinchilla Ureña ${ }^{2}$ Rosa Ibarra Ureña ${ }^{3}$ Karol Calvo Arrieta ${ }^{4}$

1, 2, y 3 Microbiólogos y Químicos Clínicos, Caja Costarricense de Seguro Social, Puntarenas, Costa Rica. 4 Microbióloga y Química Clínica, Caja Costarricense de Seguro Social, San José, Costa Rica

\section{RESUMEN}

EI SARS-CoV-2 es el coronavirus responsable de la pandemia actual, la muestra de elección para el diagnóstico de esta infección es el hisopado faríngeo. Esta muestra cuenta con varias desventajas: requiere materiales para su recolección que han escaseado a nivel mundial, su sensibilidad depende de la pericia del profesional que toma la muestra y la toma de muestra es invasiva e incómoda. Por todo esto, surge la necesidad de buscar otra muestra con suficiente sensibilidad que pueda ser empleada para el diagnóstico.

En el siguiente trabajo se realiza una revisión de la literatura disponible acerca de la idoneidad de la saliva como dicha muestra. En la mayoría de los estudios, se evidencia una alta correlación entre resultados obtenidos con saliva y los de hisopado

\section{Cómo citar:}

Orozco Barquero, A., Chinchilla Ureña, A., Ibarra Ureña, R., \& Calvo Arrieta, K. (2021). SALIVA COMO MUESTRA PARA LA DETECCIÓN DE SARSCOV-2. Revista Ciencia Y Salud, 5(2), Pág. 63-70.

Recibido: 14/ene/2021 Aceptado: 01/mar/2021 Publicado: 16/abr/2021
Palabras Clave: SARS-CoV-2, saliva, hisopado nasofaríngeo. 


\section{CIENCIA\&SALUD}

\section{ABSTRACT}

SARS-CoV-2 is the coronavirus responsible for the current pandemic, the sample of choice for the diagnostic of this infection is the nasopharyngeal swab. This sample has several disadvantages, it requires materials for its collection that have been in low supply relative to demand worldwide, its sensitivity is dependent on the expertise of the professional who takes the sample and the sampling results uncomfortable and invasive. For all this, the need arises to look for another sample with enough sensitivity that can be used for diagnosis.

In the following work, a review of the available literature about the saliva as sample for diagnosis, is carried out. In most studies, there is a high correlation between results obtained with saliva and those of nasopharyngeal swab, with viral loads high enough to be detected, especially if it is the first saliva in the morning prior to the intake of food and without having performed tooth brushing.

Keywords: SARS-CoV-2, saliva, nasopharyngeal swab.

\section{INTRODUCCIÓN:}

A finales del año 2019, la comisión de Salud de la provincia Hubei en China (Health Commission of Hubei) anunció un grupo de casos de neumonía, cuyo origen era inexplicable1. La mayoría de dichos casos, sin origen aparente, lograron ser trazados geográficamente al mercado mayorista de mariscos en Huanan1, donde se reportó la supuesta venta de carne procedente de la caza de animales1.

Mediante las técnicas de secuenciación, un betacoronavirus fue descubierto en las muestras de pacientes con neumonía2. Los coronavirus son virus ARN, que están ampliamente distribuidos entre los seres humanos, mamíferos y aves3. Estos han sido reportados como causantes de enfermedades respiratorias, entéricas, hepáticas e inclusive neurológicas en humanos4.

Hasta hace poco tiempo, solo se conocía de seis especies de esta familia que causan enfermedad en humanos5. Cuatro de estos son agentes etiológicos de síntomas de resfriado en individuos inmunocompetentes (229E, OC43, NL63 y HKU1)5,6 y los dos restantes (SARS-CoV y MERS-CoV) son de origen zoonótico y frecuentemente asociados a enfermedad fatal 6,7,8.

El betacoronavirus secuenciado a partir de las muestras de pacientes con neumonía en Wuhan es el causante de la actual pandemia2. Este fue denominado SARS-CoV-2 por el síndrome agudo respiratorio severo que provoca9. El nuevo coronavirus se transmite de forma rápida de persona a persona, lo cual le ha permitido llegar a múltiples países velozmente10.

EI SARS-CoV-2, como otros coronavirus, es un virus ARN de sentido positivo, no segmentado y de $29.9 \mathrm{~kb}$ de longitud10. Está compuesto por tres proteínas estructurales, la proteína $\mathrm{S}$ de espícula, la proteína $\mathrm{M}$ de membrana y la proteína E de envoltura y la proteína de nucleocápside N 8,11.; siendo la proteína S la res- 


\section{CIENCIA\&SALUD}

ponsable de la apariencia característica de corona y de mediar la entrada del virus a la célula, a través del receptor ACE2, que se expresa en el tejido pulmonar, cardiaco, renal y endotelio vascular 8,12,13,14.

Los primeros casos reportados de la enfermedad causada por el nuevo coronavirus o Covid-19 presentaban como síntomas más comunes: fiebre, tos, mialgia y disnea, la cual se presentó en más de la mitad de los casos15. Otros menos comunes fueron: producción de esputos, hemoptisis, dolor abdominal, vómitos y diarrea.

En adición, se ha reportado una incidencia moderada de afecciones neurológicas, manifestándose en una variedad de síntomas incluyendo anosmia, pérdida del gusto, delirios, convulsiones. La neumonía puede ocurrir en casos moderados y llegar hasta enfermedad pulmonar severa, presentando neumonía con disnea e hipoxia. Un menor porcentaje de los enfermos desarrolla enfermedad grave con fallo respiratorio y multiorgánico, llegando a requerir ser ingresados a salas de cuidados intensivos o hasta la muerte en el peor de los escenarios 16,17 .

La técnica de elección para la detección del SARS-CoV-2 es la reacción en cadena de la polimerasa con transcripción reversa en tiempo real (RT-PCR, del inglés reverse transcription- polymerase chain reaction), a partir de una muestra tomada con hisopado nasofaríngeo 18.

Cabe mencionar, que si bien el hisopado nasofaríngeo ha sido la muestra de elección y la procesada con mayor frecuencia, dado que el grueso de los casos son pacientes ambulatorios, en otros casos de pacientes con síntomas severos o inclusive niños, se recomienta recolectar espécimenes de tracto respiratorio bajo como: esputos, lavado bronqueoalveolares o aspirados traqueales 18.

La magnitud que ha alcanzado esta pandemia ha llevado a una demanda sin precedentes de los diferentes materiales involucrados en la toma de esta muestra, hisopos, tubos con medio de transporte, equipo de protección personal, entre otros, son cada vez más escasos y difíciles de adquirir19.

Por otro lado, la toma de un hisopado nasofaríngeo implica contacto directo entre los trabajadores de salud y los posibles infectados, esto incrementa el riesgo de contagio del personal, por la exposición a los aerosoles formados durante este procedimiento. Cabe resaltar que la toma de un hisopado nasofaríngeo representa un procedimiento invasivo, incómodo y que incluso podría causar sangrados de los tejidos sometidos al hisopado, especialmente en individuos trombocitopénicos20.

Ante este panorama, se hace necesaria la búsqueda de otros procedimientos de muestreo que sean seguros, que requieran menor cantidad de materiales y que idealmente sean realizados por los pacientes de forma autosuficiente.

La saliva se ha convertido entonces en una opción atractiva, pues no necesita ni equipo de toma de muestra ni medios de transporte, además puede ser recolectada por el paciente, disminuyendo el uso de equipo de protección y sin exponer a posibles contagios al personal de salud.

\section{Materiales y métodos:}

Diseño: Se realizó una revisión de la bibliografía disponible a la fecha, en la base de datos de acceso libre y especializada en ciencias de la salud PubMed.

Estrategia de búsqueda: Para la búsqueda de estudios relacionados con el tema de interés, se empleó la opción PubMed Advance Search Builder de la base de datos, con los términos SARS-CoV-2 AND Covid-19 


\section{CIENCIA\&SALUD}

AND detection AND saliva.

Criterios de inclusión y exclusión: Se tomaron en cuenta solo aquellos artículos que se pudieran accesar de manera libre y se encontraran disponibles de manera completa. Además, se delimitó el análisis a los que hubiesen sido publicados o aceptados durante el período de enero del 2020 a diciembre del 2020.

\section{DESARROLLO:}

Existe una amplia cantidad de estudios científicos destinados a comprobar la utilidad de la saliva como una muestra que sea lo suficientemente sensible, comparando la concordancia entre esta y los hisopados nasofaríngeos en la detección de este virus y diagnóstico de la enfermedad que causa.

Es importante mencionar que a la fecha se han desarrollado diversas metodologías que resultan útiles para el diagnóstico de la enfermedad, tales como: las de detección de antígenos virales, las de detección de anticuerpos contra este virus o las de detección de ARN por retro transcripción en tiempo real y amplificación en cadena de la polimerasa (RT-PCR) o amplificación isotérmica mediada por bucle de transcripción inversa. No obstante, la RT-PCR es el método actualmente más recomendado para el diagnóstico de la infección por SARS-CoV2 y es por esta razón, que en el presente trabajo revisamos literatura que compara las muestras en cuestión (hisopado nasofaríngeo y saliva) únicamente mediante dicha metología21.

En un número considerable de estudios, en los que además de tomar un hisopado nasofaríngeo se solicitó a las personas recoger una muestra de saliva, se evidencia que, a pesar de que el porcentaje de positividad es mayor en los hisopados, existe una concordancia superior al 90\% en la sensibilidad entre ambos22,23,24. De la misma manera, se demuestra que, a pesar de que las cargas virales presentes en las muestras recogidas con hisopados nasofaríngeos suelen ser mayores con respecto a la saliva, no hay diferencias significativas entre ambos tipos de muestras. Además, indican que la carga viral presente en la saliva es suficientemente superior a los límites de detección propios de cada método utilizado hasta el momento9,25,26.

Cabe recalcar que inclusive, existen estudios donde en contraste a lo anterior, se ha demostrado la existencia de una mayor carga viral en saliva que en hisopados nasofaríngeos provenientes de la misma persona y al mismo tiempo27,28,29. Además, dicha carga viral, es más alta durante la primera semana de infección, precisamente cuando la persona excreta mayor cantidad de partículas virales, que potencialmente pueden infectar a otros. Esto sugiere que la carga viral en saliva podría ser un buen predictor del potencial para transmitir el SARS-CoV-2 de los pacientes infectados30,31.

Las diferencias de la carga virales en la literatura, pueden potencialmente deberse a las formas y tiempo en el que se realiza la recolección de la saliva o a la falta de pericia con la que se toma el hisopado nasofaríngeo 32,33. Para lograr tales valores de concordancia entre los hisopados nasofaríngeos y la saliva es de gran importancia optimizar el método de recolección, pues variaciones en el mismo, han demostrado causar una menor sensibilidad a partir de este tipo de muestra28. La primera saliva de la mañana evidencia tener una mayor carga viral en pacientes hospitalizados y sintomáticos, así como en tamizajes en la población9,28. A pesar de que la mayoría de estudios han sido realizados en pacientes hospitalizados y con diagnósticos confirmados, resultados similares se han obtenido de personas no hospitalizadas e incluso sin diagnóstico confirmado, lo que convierte a la saliva en una muestra muy útil en tamizajes a gran escala, tanto en adultos como en población pediátrica 23,25,28.

En la mayoría de estudios en donde la saliva mostró ser un espécimen lo suficientemente sensible, similar a los hisopados nasofaríngeos, un factor en común fue el solicitar que la muestra de saliva fuera recogida luego de toser o expectorar, para asegurarse que se incluya también la saliva presente en la garganta, y no 


\section{CIENCIA\&SALUD}

solamente la presente en la cavidad bucal. Se indica además, que no se ingieran alimentos ni se realice lavado de dientes antes de llevar a cabo lo anterior, que se debe hacer en repetidas veces hasta obtener más de un mililitro de saliva 9,24,25,28. Dicha muestra puede ser recolectada en frasco estéril, similar a los que se usan para recoger esputos y ser enviada en hielera o cualquier método de refrigeración al laboratorio donde será analizada sin que se afecte la estabilidad 24,34.

Es justo mencionar que hay condiciones propias de la saliva que pueden afectar la realización de la prueba de detección. La principal es la viscosidad aumentada en algunas muestras, que puede ocasionar errores en las determinaciones debido a atascos en los instrumentos, por lo que en algunas ocasiones se hace necesario homogenizar en un vórtex o centrifugar la muestra para disminuir el porcentaje de muestras inválidas 9,24 . Aún con estas medidas correctivas adicionales, la tasa de muestras de saliva inválidas para ser analizadas es mayor que las que se presentan por medio de hisopado nasofaríngeo 25,35.

\section{CONCLUSIONES}

Ante el panorama actual y apoyado en el alto número de estudios que han tenido resultados favorables, la saliva, siempre que sea recolectada de una forma óptima y tratada correctamente previo a su procesamiento, es una muestra prometedora para la detección del SARS-CoV-2. Lo anterior debido a su bajo costo, a que no requiere de materiales para la toma de muestra y a que puede ser recolectada por las personas sin involucrar al personal de salud o emplear un método invasivo e incómodo, lo que a la postre disminuye la demanda de insumos que resultan escasos y sobre todo, las posibilidades de contagio del recurso humano capacitado en salud.

\section{REFERENCIAS BIBLIOGRÁFICAS}

1. Chan JF, Yuan S, Kok KH, To KK, Chu H, Yang J, Xing F, Liu J, Yip CC, Poon RW, Tsoi HW, Lo SK, Chan KH, Poon VK, Chan WM, Ip JD, Cai JP, Cheng VC, Chen H, Hui CK, Yuen KY. A familial cluster of pneumonia associated with the 2019 novel coronavirus indicating person-to-person transmission: a study of a family cluster. Lancet. 2020 Feb 15;395(10223):514-523.

2. Zhu N, Zhang D, Wang W, Li X, Yang B, Song J, Zhao X, Huang B, Shi W, Lu R, Niu P, Zhan F, Ma X, Wang D, Xu W, Wu G, Gao GF, Tan W; China Novel Coronavirus Investigating and Research Team. A Novel Coronavirus from Patients with Pneumonia in China, 2019. N Engl J Med. 2020 Feb 20;382(8):727-733.

3. Weiss SR, Leibowitz JL. Coronavirus pathogenesis. Adv Virus Res 2011; 81:85-164.

4. Masters PS, Perlman S. Coronaviridae. En: Knipe DM, Howley PM, eds. Fields virology. 6th ed. Lippincott Williams \& Wilkins, 2013:825-858.

5. Su, S., Wong, G., Shi, W., Liu, J., Lai, A., Zhou, J., Liu, W., Bi, Y. and Gao, G., Epidemiology, genetic recombination, and pathogenesis of coronaviruses. Trends Microbiol 2016; 24:490-502.

6. Oves M, Ravindran M, Rauf MA, Omaish Ansari M, Zahin M, Iyer AK, Ismail IMI, Khan MA, Palaniyar N. Comparing and Contrasting MERS, SARS-CoV, and SARS-CoV-2: Prevention, Transmission, 


\section{CIENCIA\&SALUD}

Management, and Vaccine Development. Pathogens. 2020 Nov 26;9(12):985.

7. Rabaan, A.A.; Al-Ahmed, S.H.; Haque, S.; Sah, R.; Tiwari, R.; Malik, Y.S.; Dhama, K.; Yatoo, M.I.; Bonilla-Aldana, D.K.; Rodriguez-Morales, A.J. SARS-CoV-2, SARS-CoV, and MERS-COV: A comparative overview. Infez. Med. 2020, 28, 174-184.

8. Cui J, Li F, Shi ZL. Origin and evolution of pathogenic coronaviruses. Nat Rev Microbiol 2019; 17:181-192.

9. Procop GW, Shrestha NK, Vogel S, Van Sickle K, Harrington S, Rhoads DD, Rubin BP, Terpeluk P. A Direct Comparison of Enhanced Saliva to Nasopharyngeal Swab for the Detection of SARSCoV-2 in Symptomatic Patients. J Clin Microbiol. 2020 Oct 21; 58(11):e01946-20.

10. Wang P, Anderson N, Pan Y, Poon L, Charlton C, Zelyas N, Persing D, Rhoads D, Babcock H. 2020. The SARS-CoV-2 outbreak: diagnosis, infection prevention, and public perception. Clin Chem. 2020 Mar: 10:hvaa080.

11. Wu, J.T.; Leung, K.; Leung, G.M. Nowcasting and forecasting the potential domestic and international spread of the 2019-nCoV outbreak originating in Wuhan, China: A modelling study. Lancet. 2020:395: 689-697.

12. Walls, A.C.; Park, Y.J.; Tortorici, M.A.;Wall, A.; McGuire, A.T.; Veesler, D. Structure, function, and antigenicity of the SARS-CoV-2 spike glycoprotein. Cell. 2020:181:81-292.e286.

13. Luan, J.; Lu, Y.; Jin, X.; Zhang, L. Spike protein recognition of mammalian ACE2 predicts the host rangeand an optimized ACE2 for SARS-CoV-2 infection. Biochem. Biophys. Res. Commun. 2020: 526: 165-169.

14. Andersen, K.G.; Rambaut, A.; Lipkin,W.I.; Holmes, E.C.; Garry, R.F. The proximal origin of SARSCoV-2. Nat. Med. 2020. 26: 450-452.

15. Huang C, Wang Y, Li X, Ren L, Zhao J, Hu Y, Zhang L, Fan G, Xu J, Gu X, Cheng Z, Yu T, Xia J, Wei Y, Wu W, Xie X, Yin W, Li H, Liu M, Xiao Y, Gao H, Guo L, Xie J, Wang G, Jiang R, Gao Z, Jin Q, Wang J, Cao B. Clinical features of patients infected with 2019 novel coronavirus in Wuhan, China. Lancet. 2020 Feb 15;395(10223):497-506.

16. Mao L, Jin H, Wang M, Hu Y, Chen S, He Q, Chang J, Hong C, Zhou Y, Wang D, Miao X, Li Y, Hu B. Neurologic Manifestations of Hospitalized Patients With Coronavirus Disease 2019 in Wuhan, China. JAMA Neurol. 2020 Jun 1;77(6):683-690.

17. Wu Z, McGoogan JM. Characteristics of and Important Lessons From the Coronavirus Disease 2019 (COVID-19) Outbreak in China: Summary of a Report of 72314 Cases From the Chinese Center for Disease Control and Prevention. JAMA. 2020 Apr 7;323(13):1239-1242. 


\section{CIENCIA\&SALUD}

18. Hong KH, Lee SW, Kim TS, Huh HJ, Lee J, Kim SY, Park JS, Kim GJ, Sung H, Roh KH, Kim JS, Kim HS, Lee ST, Seong MW, Ryoo N, Lee H, Kwon KC, Yoo CK. Guidelines for Laboratory Diagnosis of Coronavirus Disease 2019 (COVID-19) in Korea. Ann Lab Med. 2020 Sep;40(5):351-360.

19. Ranney ML, Griffeth V, Jha AK. Critical Supply Shortages - The Need for Ventilators and Personal Protective Equipment during the Covid-19 Pandemic. N Engl J Med. 2020 Apr 30;382(18):e41.

20. Nagura-Ikeda M, Imai K, Tabata S, Miyoshi K, Murahara N, Mizuno T, Horiuchi M, Kato K, Imoto Y, Iwata M, Mimura S, Ito T, Tamura K, Kato Y. Clinical Evaluation of Self-Collected Saliva by Quantitative Reverse Transcription-PCR (RT-qPCR), Direct RT-qPCR, Reverse Transcription-Loop-Mediated Isothermal Amplification, and a Rapid Antigen Test To Diagnose COVID-19. J Clin Microbiol. 2020 Aug 24;58(9):e01438-20.

21. Sueki A, Matsuda K, Yamaguchi A, Uehara M, Sugano M, Uehara T, Honda T. 2016. Evaluation of saliva as diagnostic materials for influenza virus infection by PCR-based assays. Clin Chim Acta 453:71-4.

22. Williams E, Bond K, Zhang B, Putland M, Williamson DA. Saliva as a Noninvasive Specimen for Detection of SARS-CoV-2. J Clin Microbiol. 2020 Jul 23;58(8):e00776-20.

23. Yee R, Truong T, Pannaraj PS, Eubanks N, Gai E, Jumarang J, Turner L, Peralta A, Lee Y, Dien Bard J. Saliva is a promising alternative specimen for the detection of SARS-CoV-2 in children and adults. J Clin Microbiol. 2020 Nov 25:JCM.02686-20.

24. Azzi L, Carcano G, Gianfagna F, Grossi P, Gasperina DD, Genoni A, Fasano M, Sessa F, Tettamanti L, Carinci F, Maurino V, Rossi A, Tagliabue A, Baj A. Saliva is a reliable tool to detect SARSCoV-2. J Infect. 2020 Jul;81(1):e45-e50.

25. Kandel C, Zheng J, McCready J, Serbanescu MA, Racher H, Desaulnier M, Powis JE, Vojdani K, Finlay L, Sheldrake E, Vermeiren C, Katz K, McGeer A, Kozak R, Goneau LW. Detection of SARS-CoV-2 from Saliva as Compared to Nasopharyngeal Swabs in Outpatients. Viruses. 2020 Nov 17;12(11):1314.

26. Landry ML, Criscuolo J, Peaper DR. Challenges in use of saliva for detection of SARS CoV-2 RNA in symptomatic outpatients. J Clin Virol. 2020 Sep;130:104567.

27. Wyllie AL, Fournier J, Casanovas-Massana A, Campbell M, Tokuyama M, Vijayakumar P, Warren JL, Geng B, Muenker MC, Moore AJ, Vogels CBF, Petrone ME, Ott IM, Lu P, Venkataraman A, Lu-Culligan A, Klein J, Earnest R, Simonov M, Datta R, Handoko R, Naushad N, Sewanan LR, Valdez J, White EB, Lapidus S, Kalinich CC, Jiang X, Kim DJ, Kudo E, Linehan M, Mao T, Moriyama M, Oh JE, Park A, Silva J, Song E, Takahashi T, Taura M, Weizman OE, Wong P, Yang Y, Bermejo S, Odio CD, Omer SB, 


\section{CIENCIA\&SALUD}

Dela Cruz CS, Farhadian S, Martinello RA, Iwasaki A, Grubaugh ND, Ko Al. Saliva or Nasopharyngeal Swab Specimens for Detection of SARS-CoV-2. N Engl J Med. 2020 Sep 24;383(13):1283-1286.

28. Fakheran O, Dehghannejad M, Khademi A. Saliva as a diagnostic specimen for detection of SARS-CoV-2 in suspected patients: a scoping review. Infect Dis Poverty. 2020 Jul 22;9(1):100.

29. Sakanashi D, Asai N, Nakamura A, Miyazaki N, Kawamoto Y, Ohno T, Yamada A, Koita I, Suematsu H, Hagihara M, Shiota A, Kurumiya A, Sakata M, Kato S, Muramatsu Y, Koizumi Y, Kishino T, Ohashi W, Yamagishi Y, Mikamo H. Comparative evaluation of nasopharyngeal swab and saliva specimens for the molecular detection of SARS-CoV-2 RNA in Japanese patients with COVID-19. J Infect Chemother. 2021 Jan;27(1):126-129.

30. Iwasaki S, Fujisawa S, Nakakubo S, Kamada K, Yamashita Y, Fukumoto T, Sato K, Oguri S, Taki K, Senjo H, Sugita J, Hayasaka K, Konno S, Nishida M, Teshima T. Comparison of SARS-CoV-2 detection in nasopharyngeal swab and saliva. J Infect. 2020 Aug;81(2):e145-e147.

31. Jamal AJ, Mozafarihashjin M, Coomes E, Powis J, Li AX, Paterson A, Anceva-Sami S, Barati S, Crowl G, Faheem A, Farooqi L, Khan S, Prost K, Poutanen S, Taylor M, Yip L, Zhong XZ, McGeer AJ, Mubareka S; Toronto Invasive Bacterial Diseases Network COVID-19 Investigators. Sensitivity of nasopharyngeal swabs and saliva for the detection of severe acute respiratory syndrome coronavirus 2 (SARS-CoV-2). Clin Infect Dis. 2020 Jun 25:ciaa848.

32. Hanson KE, Barker AP, Hillyard DR, Gilmore N, Barrett JW, Orlandi RR, Shakir SM. Self-Collected Anterior Nasal and Saliva Specimens versus Health Care Worker-Collected Nasopharyngeal Swabs for the Molecular Detection of SARS-CoV-2. J Clin Microbiol. 2020 Oct 21;58(11):e01824-20.

33. Abasiyanik MF, Flood B, Lin J, Ozcan S, Rouhani SJ, Pyzer A, Trujillo J, Zhen C, Wu P, Jumic S, Wang A, Gajewski TF, Wang P, Hartley M, Ameti B, Niemiec R, Fernando M, Aydogan B, Bethel C, Matushek S, Beavis KG, Agrawal N, Segal J, Tay S, Izumchenko E. Sensitive detection and quantification of SARS-CoV-2 in saliva. medRxiv [Preprint]. 2020 Dec 7:2020.12.04.20241059.

34. Ott IM, Strine MS, Watkins AE, Boot M, Kalinich CC, Harden CA, Vogels CBF, Casanovas-Massana A, Moore AJ, Muenker MC, Nakahata M, Tokuyama M, Nelson A, Fournier J, Bermejo S, Campbell M, Datta R, Dela Cruz CS, Farhadian SF, Ko Al, Iwasaki A, Grubaugh ND, Wilen CB, Wyllie AL. Simply saliva: stability of SARS-CoV-2 detection negates the need for expensive collection devices. medRxiv [Preprint]. 2020 Aug 4:2020.08.03.20165233.

35. Chen JH, Yip CC, Poon RW, Chan KH, Cheng VC, Hung IF, Chan JF, Yuen KY, To KK. Evaluating the use of posterior oropharyngeal saliva in a point-of-care assay for the detection of SARS-CoV-2. Emerg Microbes Infect. 2020 Dec;9(1):1356-1359. 